\title{
Onkologische Pflege braucht gute Konzepte
}

\section{Onkologische Zertifizierungen, Pflegekonzepte und Pflegeberatung Onkologische} Zertifizierungen zielen darauf, die Behandlung onkologischer Patienten so zu verbessern, dass sie eine Behandlung von höchster Qualität erfahren. Für die onkologische Pflege sind bisher jedoch nur Qualitätskriterien formuliert, aus denen sich vielfältige Interpretationsmöglichkeiten ergeben. Ziel sollte deshalb ein einheitliches Verständnis mit eindeutig formulierten Konzepten und Aufgaben sein.

Susanne Kelber

\section{ZUSAMMENFASSUNG}

Trotz zahlreicher onkologischer Zertifizierungen gibt es für Pflegende bisher keine einheitliche Definition eines onkologischen Pflegekonzeptes und einer onkologischen Pflegeberatung. Daraus ergeben sich vielfältige Interpretationsmöglichkeiten der Vorgaben mit entsprechenden heterogenen Ergebnissen. Ein wesentliches Ziel ist es, ein einheitliches Verständnis von einem onkologischen Pflegekonzept zu schaffen. Als wichtigste Voraussetzung für ein entsprechendes Konzept ist die Ziel- und Zweckklärung. Danach sollte die Erstellung methodengeleitet erfolgen. Onkologische Pflegekonzepte sollen eine Chance für die onkologische Pflege darstellen, ihre Leistungsfähigkeit und den Nutzen für die Interessensgruppen transparent zu machen. Ein möglicher Inhalt ist beispielsweise die onkologische Pflegeberatung. Für Patienten ist eine strukturierte, individuelle Beratung eine der wirksamsten Unterstützungsmöglichkeiten für das Selbstmanagement.

Schlüsselwörter: Zertifizierung, onkologische Pflege, Pflegekonzept, Pflegeberatung, Konzepterstellung

D ie von der Deutschen Krebsgesellschaft (DKG) initiierten onkologischen Zertifizierungen sollen die Behandlung onkologischer Patienten so verbessern, dass diese in jeder Phase ihrer Erkrankung eine Behandlung von höchster Qualität erfahren. Den Anfang machte die DKG mit der Gesellschaft für Senologie, als sie die Anforderungen für eine Zertifizierung von Brustkrebszentren erarbeitete. Seit 2003 werden Brustkrebszentren zertifiziert, im Laufe der Jahre kamen weitere Zentren dazu (Abb.
1, e-only). Das von der DKG beauftragte unabhängige Institut OnkoZert betreut für das Zertifizierungssystem die Überprüfung von Organkrebszentren und onkologischen Zentren gemäß den entsprechenden fachlichen Anforderungen.

\section{Onkologische Pflege hat Einfluss auf die Zertifizierungen}

Die zertifizierten Zentren müssen in regelmäßigen Audits nachweisen, dass sie die fachlichen Anforderungen für die Behandlung einer Tumorerkrankung erfüllen. Dabei spielt die interdisziplinäre und berufsgruppenübergreifende Zusammenarbeit aller Fachdisziplinen eine große Rolle. Grundlage für die Zertifizierungen ist seit 2003 ein Erhebungsbogen, in dem fachliche Anforderungen abgebildet sind, die bei der Zertifizierung erfüllt werden müssen (DKG 2019). So heißt es in den „Fachlichen Anforderungen Onkologische Zentren“(FAO) unter Punkt 1.8 Pflege - Zuständigkeiten/ Aufgaben unter anderem, dass patientenbezogene Aufgaben wie die kontinuierliche Information und Beratung des Patienten (und deren Angehörige) während des gesamten Krankheitsverlaufes sowie die Durchführung, Koordination und der Nachweis von strukturierten Beratungsgesprächen und Anleitung von Patienten und Angehörigen Teil der Behandlung sein müssen. $\mathrm{Zu}$ den übergeordneten Tätigkeiten zählen die Entwicklung und Umsetzung eines Pflegekonzeptes, in dem die organspezifischen Besonderheiten der onkologischen Pflege in den Organkrebszentren/Modulen berücksichtigt werden (OnkoZert 2019).

Viele Zentren stellen diese Punkte vor große Herausforderungen. Die Konferenz onkologischer Kranken- und Kinderkrankenpflege (KOK) als eine der größten Arbeitsgruppen in der DKG macht über die Zertifizierungs-AG Eingaben in die Zertifizierungskommissionen der DKG. Die Zertifizierungs-AG besteht aus Pflegenden, Fachpflegenden und onkologisch Fachpflegenden, die die onkologische Pflege betreffenden Eingaben erarbeiten. Im nächsten Schritt werden diese Eingaben in den Zertifizierungskommissionen besprochen und verabschiedet. An diesen Sitzungen nimmt 


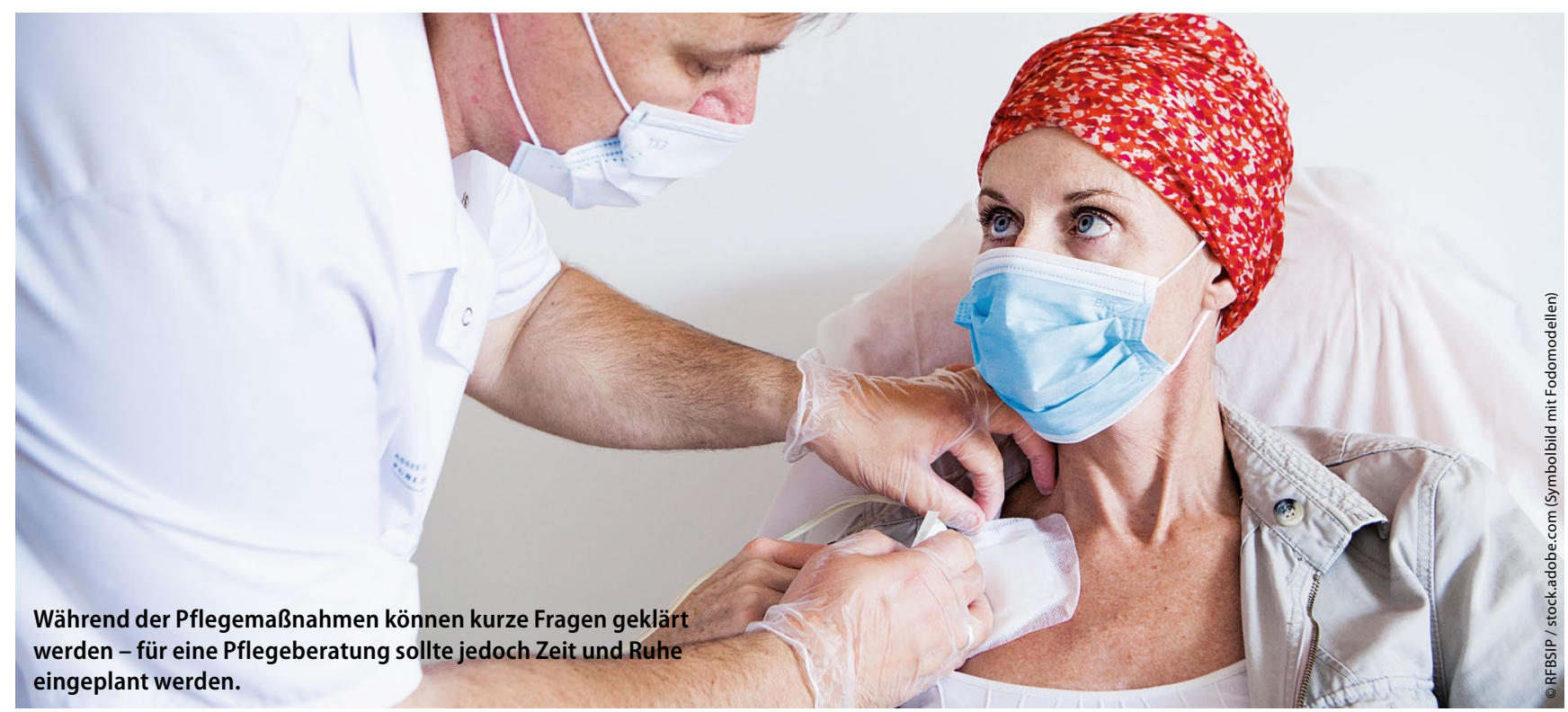

ein Vertreter der KOK teil. Letztlich werden die Änderungen im aktualisierten Erhebungsbogen sichtbar. Die onkologische Pflege bestimmt demnach in hohem Maße selbst mit, was für Zertifizierungen als Qualitätsmerkmale definiert werden.

\section{Onkologische Pflegekonzepte bilden die Leistungsfähigkeit ab}

Viele onkologische Pflegekonzepte wurden erst nach Veröffentlichung der OnkoZert-Zertifizierungskriterien erstellt. Dabei sind überall dort, wo Bestehendes oder Neuentstehendes bearbeitet wird, hierarchieübergreifende Konzepte erforderlich. Der Landespflegeausschuss Brandenburg (2016) hat es in seinem Leitfaden zum Erstellen eines Einrichtungs- und Pflegekonzeptes für vollstationäre Pflegeeinrichtungen passend formuliert: „Konzeptionslosigkeit ist Orientierungslosigkeit“. Dabei kann konzeptionelles Arbeiten Mitarbeiter motivieren, sich über das normale Maß hinaus zu engagieren, Veränderungsprozesse zu ihrer eigenen Sache zu machen und diese aktiv voranzutreiben (Ischebeck 2017).

Für die onkologische Fachpflege kann ein gutes onkologisches Pflegekonzept die Möglichkeit bieten, die Fachexpertise, den Nutzen für Patienten und Einrichtung sowie die Weiterentwicklung transparent zu machen (Kelber 2019). Onkologische Pflegekonzepte bieten die Chance, onkologische Pflege in ihrer gesamten Leistungsfähigkeit darzustellen. Professionelle onkologische Pflege bedeutet bestmögliche Behandlungs- und Betreuungsergebnisse sowie eine bestmögliche Lebensqualität in allen Phasen des Lebens bis zum Tod der zu betreuenden Menschen (Spichiger et al. 2006). Damit ein onkologisches Pflegekonzept gelingen kann, muss es Wegweiser für die Interessengruppen sein und eine sich weiterentwickelnde Herangehensweise für methodisches Arbeiten bieten. Deshalb gilt es zunächst gut zu überlegen, zu welchem Zweck das Konzept erstellt werden soll. Für die Erstellung selbst empfiehlt sich ein prinzipien-und methodengeleitetes Vorgehen. Konzepte, die Aussicht auf eine erfolgreiche Umsetzung haben, folgen dem ZEB-
RA-Prinzip (Ischebeck 2017): Zielorientiert, Empfängerorientiert Beherzt auf den Punkt gebracht, Realistisch geplant, Auslöser für Aktivitäten.

Ziele sollten gemeinsam und präzise herausgearbeitet werden und das Konzept empfängerorientiert nachvollziehbar sein. Einfache, verständliche Formulierungen und eine Gliederung machen das Konzept interessanter. „Realistisch geplant“ meint eine angemessene Zeitplanung für die Erstellung und die Umsetzung der Lösungsvorschläge. „Auslöser für Aktivitäten“ meint hier konkrete Empfehlungen für Handlungen und Strategien (Ischebeck 2017). Ein Problem der Erstellung von onkologischen Pflegekonzepten kann sein, dass die Aktivitäten aufgrund der Komplexität in unterschiedliche Richtungen gelenkt und mehrere Schwierigkeiten gleichzeitig bewältigt werden müssen. Folgende Konzeptphasen geben Orientierung für eine erfolgreiche Vorgehensweise:

— Auftrags- und Zielklärung

_ Informationen recherchieren und organisieren

- Ideen und Lösungen entwickeln

_ Das Konzept schlüssig strukturieren

- Das Konzept überzeugend schriftlich darstellen

- Das Konzept erfolgreich kommunizieren (Ischebeck 2017)

\section{Konzepte müssen gelebt und weiterentwickelt werden}

Laut Erhebungsbogen soll ein onkologisches Pflegekonzept entwickelt werden, in dem die organspezifischen Besonderheiten der onkologischen Pflege in den Organkrebszentren/Modulen Berücksichtigung finden (OnkoZert 2019). Diese Vorgabe kann unterschiedlich aufgefasst werden und erfordert aufgrund dessen vor der eigentlichen Konzepterstellung ein gemeinsames Verständnis der Ausgangssituation und eine Zielklärung aller an der Erstellung Beteiligten (Pflegemanagement, Einrichtungsleitung, Klinikdirektoren). Schon im Vorfeld ist zu überlegen, für welchen Zweck (z.B. interne Arbeitsanweisung, Patientenbroschüre, Tätigkeitsbeschreibung, Wegweiser) das Konzept stehen soll und welche Ressourcen 
(materiell, personell) dafür bereitgestellt werden müssen. Ein onkologisches Pflegekonzept nur für Zertifizierungen zu erstellen, damit es danach wieder in einer Schublade ruht, ist Ressourcenverschwendung. Onkologische Pflegekonzepte sollen gelebt werden und sich kontinuierlich weiterentwickeln (Kelber 2019). Sie sollen praxisbezogen ein „Handlungsmodell, in welchem die Ziele, die Inhalte, die Methoden und die Verfahren in einen sinnhaften Zusammenhang gebracht sind" (Landespflegeausschuss Brandenburg) bieten. Aus diesem Grund hat der KOK-Vorstand eine Empfehlung möglicher Gliederungspunkte (Tab. 1, e-only) eines onkologischen Pflegekonzeptes erarbeitet (Kelber 2019).

Nachdem die Inhalte eines Pflegekonzept festgelegt sind, gilt es dieses überzeugend zu verschriftlichen. Nachhaltige Überzeugung entsteht, wenn Menschen den Argumenten und der Gedankenführung folgen können. Das Hamburger Verständlichkeitskonzept (PCS Akademie 2018) beschreibt vier Kriterien, die verständliche Texte ausmachen: Einfachheit, Gliederung, Prägnanz und Anregung. Konzepte werden meist in intensiver Auseinandersetzung mit dem Thema erstellt. Dabei geraten die Menschen, die das Konzept letztlich umsetzen sollen, leicht aus dem Blickfeld. Um dem Elfenbeinturmeffekt entgegenzuwirken, muss der Praxisbezug hergestellt und aufrechterhalten werden. Es gilt, die Personen einzubeziehen, die das Konzept umsetzen müssen. Dafür ist eine taktisch wohlüberlegte und grundsätzlich transparente Kommunikation hilfreich. Onkologische Pflegekonzepte können also vielmehr sein, als ein durch die Zertifizierung entstandenes „Papier“. Sie können eine Chance für onkologische (Fach-)Pflegende sein, nach ihrer Qualifikation eingesetzt zu werden und Transparenz für Interessensvertreter schaffen.

\section{Onkologische Pflegeberatung ist die Basis der Patientenedukation}

Eine Krebserkrankung verändert das gesamte Leben. Die existenzbedrohende Diagnose, der eigene Rollenverlust und die Fremdbestimmung durch die Behandler bedingen ein professionelles Tun der Akteure, um Patienten Sicherheit zu geben (Pfeifer-Rabe, Hojdelewicz \& Durchschlag 2019). Patientenedukation wird als Oberbegriff für Information, Schulung/Anleitung und Beratung (AbtZegelin 2002) verstanden. Ziel ist es, Patienten darin zu unterstüt-

\section{Nitite \\ D BUCHTIPP Das Blaue Buch \\ M. Engelhardt, R. Mertelsmann, J. Duyster (Hrsg.) Das blaue Buch \\ Chemotherapie-Manual Hämatologie und Onkologie - Bewährte Behandlungsprotokolle zur optimalen Versorgung von Tumorpatienten für Ärzte, Pflegepersonal und Apotheker \\ Springer 2020 \\ ISBN 978-3-662-60380-2; 71,30€}

zen, sich neues Wissen, Handlungskompetenzen und Bewältigungsstrategien zu erarbeiten (Hacker, Slobodenka \& Titzer 2012). Patienten mit einer onkologischen Diagnose und der daraus folgenden Therapie beschreiben erfahrungsgemäß den Kontrollverlust über ihr Leben als zentrales Problem. Beim Erarbeiten von Coping-Strategien (Bewältigungsstrategien für Belastungen, neue Anforderungen und Krisen) benötigen onkologische Patienten Unterstützung, denn sie verhalten sich gerade am Anfang der Erkrankung eher passiv (Raschper 2015). Empowerment mit Selbstbefähigung, Autonomie und Selbstwirksamkeit ist ein sinnvolles Konzept, das Patienten helfen kann, sich aktiv zu beteiligen. Gleichzeitig wirkt es dem Gefühl des Ausgeliefertseins entgegen. Dabei wird auf Selbsthilfekompetenzen, Potenziale und persönliche Ressourcen zurückgegriffen (Kanfer, Reinecker \& Schmelzer 2012).

Die Zertifizierungskriterien benennen die Durchführung und den Nachweis strukturierter Beratungsgespräche (s.o.). Situierte ad hoc Beratungen finden erfahrungsgemäß oft neben den Pflegetätigkeiten statt. Davon zu unterscheiden sind terminierte, strukturierte Beratungsgespräche, die einem methodischem Vorgehen folgen (z.B. Pflegeberatungssprechstunden in einem geschützten Rahmen). Beratung als Prozess meint sowohl mehrere aufeinander folgende Gespräche als auch den prozesshaften Charakters eines einzelnen Gespräches. Für die Pflegeberatung lässt sich ein am Pflegeprozess orientiertes Phasenmodell als sinnvolles Gliederungsinstrument ableiten (Hummel-Gaatz \& Doll 2007). Gespräche machen einen Großteil der pflegerischen Arbeit aus, werden aber aufgrund ihres Aufwands selten korrekt dokumentiert und finden in der Personalbemessung keine zeitliche Berücksichtigung (Hacker, Slobodenka \& Titzer 2012).

\section{Gute Pflegeberatung schafft Entlastung beim Patienten}

Der Beratungsbedarf äußert sich bei onkologischen Patienten durch die lebensbedrohliche Diagnose und die damit entstandenen Veränderungsprozesse (Dokken, Stukenkemper, Huber \& ThokeColberg 2005). Das Bedürfnis der Patienten nach behandlungsbezogenen Informationen (van Weert, Bolle, van Dulmen \& Jansen 2013) und der Kommunikation von möglichen Nebenwirkungen (van Weert et al. 2009) bildet den Schwerpunkt in onkologischen Pflegeberatungen. Daher greifen onkologische Fachpflegende im Rahmen von edukativen Beratungsgesprächen das Supportiv- und Nebenwirkungsmanagement als Schwerpunkt auf (Mattern, Feyerherd \& Luntz 2017). Weitere Gesprächsinhalte sind die veränderten Lebensumstände mit körperlichen und emotionalen Belastungen durch Einschränkungen im Alltag, Ängste und Krankheitsverarbeitung (Evers 2015). Es gilt, die Ratsuchenden in ihrer Wahrnehmung der Veränderungen zu unterstützen (Spee 2014). Die klientenzentrierte, nicht-direktive Gesprächsführung nach Carl Rogers dient als Gesprächsansatz für die Gesprächsführung. Ressourcenidentifizierung und Entwicklung von eigenen Lösungsmöglichkeiten stehen unabhängig vom Beratenden im Fokus (Reibnitz, Sonntag \& Strackbein 2017). Dabei werden nach Rogers an den Berater Kernbedingungen gestellt: Kongruenz in der Beziehung, bedingungslose positive Wertschätzung und empathisches Verständnis (Maltby, Day \& Macaskill 2011). Die Studienlage im Bereich der Symptomkontrolle und der Adhärenzforschung ist mittlerweile umfangreich (Baumann \& Welslau 2017). 


\section{PFLEGE PRAXIS}

Dass onkologische Pflegeinterventionen durch eine Beratung einen positiven Effekt auf die Symptombelastung der Patienten haben, ist ein Zeichen für die Wirksamkeit der Pflegeberatung (Coolbrandt et al. 2014). Das Gefühl unterstützt zu werden und sich fachkundig beraten zu lassen, gab den Patienten das Gefühl, beruhigt und besser in der Lage zu sein, mit ihren Symptomen umzugehen (Coolbrandt et al. 2018). Es wurde aber auch gezeigt, dass es Unterschiede in der Pflegequalität gibt, die einen Einfluss auf die Symptomlast der Patienten hat (Armes et al. 2014). Da sehr offen ist, was unter Beratungsqualität zu verstehen ist, wurden für die ambulante Beratung nach $\$ 7$ a SGB XI Qualitätsdimensionen bezeichnet, welche die Bereiche Lösungsqualität, Beziehungsqualität, Methodenqualität (strukturierte Gesprächsführung), Informationsqualität und Zugangsqualität umfassen (Michell-Auli \& Gerlich 2018).

! FAZIT

Onkologische Pflegekonzepte, die nachhaltig gelebt werden, können wertschöpfend sein für die Pflegenden, für das Pflegemanagement, für andere Professionen, für die Einrichtung und nicht zuletzt für die Patienten.

Die Wirksamkeit von onkologischen Pflegeberatungen ist im Alltag spürbar und leistet einen wichtigen Beitrag zum Nationalen Krebsplan.

Mit dem antizipierten Fachwissen nehmen die onkologischen Fachpflegenden eine wichtige Schlüsselfunktion im multidisziplinären Team ein.

\section{Hier steht eine Anzeige.}

$\downarrow$ Die Literaturliste erhalten Sie online über das eMagazin der PflegeZeitschrift und auf springerpflege.de

A Abb. 1 "Zertifizierte onkologische Zentren“ erhalten Sie online über das eMagazin der PflegeZeitschrift und auf springerpflege.de

\ab. 1 „Inhalte onkologisches Pflegekonzept " erhalten Sie online über das eMagazin der PflegeZeitschrift und auf springerpflege.de

\section{Autorenkontakt:}

Susanne Kelber B.A. ist Onkologische Fachpflegerin, Medizinpädagogin, Dozentin und Referentin am Universitätsklinikum Frankfurt und im Vorstand der Konferenz Onkologischer Kranken- und Kinderkrankenpflege (KOK) in der Deutschen Krebsgesellschaft e.V.

E-Mail: susanne.kelber@gmail.com 